City University of New York (CUNY)

CUNY Academic Works

\title{
Species Identification of Necrophagous Insect Eggs Based on Amino Acid Profile Differences Revealed by Direct Analysis in Real Time-High Resolution Mass Spectrometry
}

Justine E. Giffen

University at Albany, State University of New York

Jennifer Y. Rosati

CUNY John Jay College

Cameron M. Longo

University at Albany, State University of New York

Rabi A. Musah

University at Albany, State University of New York

\section{How does access to this work benefit you? Let us know!}

More information about this work at: https://academicworks.cuny.edu/jj_pubs/209

Discover additional works at: https://academicworks.cuny.edu

This work is made publicly available by the City University of New York (CUNY).

Contact: AcademicWorks@cuny.edu 


\title{
Species Identification of Necrophagous Insect Eggs Based on Amino Acid Profile Differences Revealed by Direct Analysis in Real Time- High Resolution Mass Spectrometry
}

\author{
Justine E. Giffen, ${ }^{\dagger}$ Jennifer Y. Rosati, ${ }^{\ddagger}$ Cameron M. Longo, ${ }^{\dagger}$ and Rabi A. Musah ${ }^{*}{ }^{\dagger}$ (i)
}

${ }^{\dagger}$ Department of Chemistry, University at Albany, State University of New York, 1400 Washington Avenue, Albany, New York 12222, United States

${ }^{\ddagger}$ John Jay College of Criminal Justice, 524 West 59th Street, New York, New York 10019, United States

\section{Supporting Information}

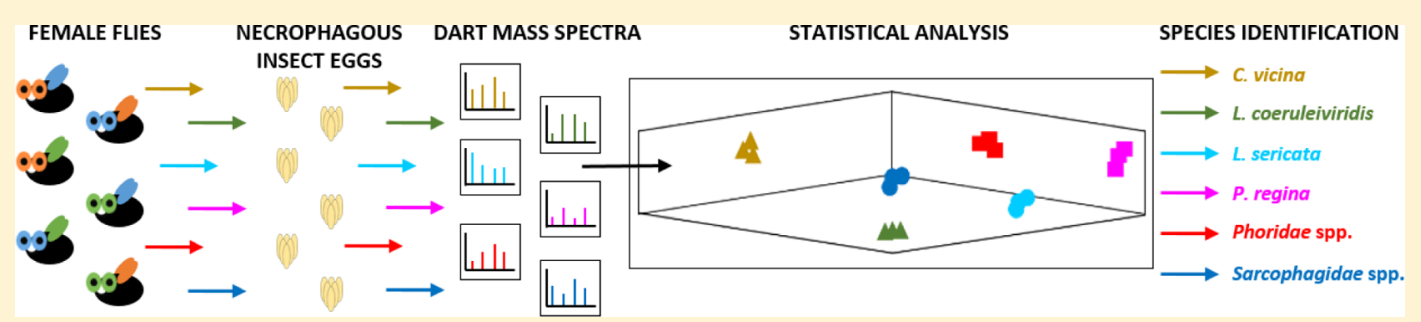

ABSTRACT: The colonization of decomposing remains by necrophagous insects such as blow flies is of forensic importance because the progression through the various stages of insect development can be correlated to time of death. The ability to infer this information hinges on accurate determination of the fly species that are associated with the entomological evidence collected. This evidence can include eggs, larvae, pupae, and puparial casings. Determination of the egg's identity is particularly challenging because the eggs of multiple species are morphologically very similar. We report here that the species identity of fly eggs can be determined from their chemical fingerprint signatures acquired by direct analysis in real time high-resolution mass spectrometry (DART-HRMS). Thus, freshly laid eggs were collected and readily distinguished from multiple necrophagous fly species in the Manhattan area of New York City. These species included representatives from the blow fly family (Calliphoridae), specifically Calliphora vicina, Lucilia sericata, L. coeruleiviridis, and Phormia regina species as well as the Phoridae and Sarcophagidae families. Multivariate statistical analysis of their observed DART-HRMS spectra revealed intraspecies similarities and interspecies differences that were the basis of species differentiation. The chemical basis of discrimination was differences in amino acid profiles. This represents the first chemically based fly egg identification approach with applications to forensic entomology. The rapidity of the method makes feasible the generation of a fly egg chemical profile database against which the DART-HRMS spectra of unknown eggs can be screened to rapidly assess species identity without needing to rear the eggs to adulthood.

$I^{t}$

$\mathrm{t}$ has long been recognized that colonization of corpses by various insect types can provide critical information about the circumstances under which a death occurred. ${ }^{1,2}$ In this regard, the utility of "carrion insects" such as those of the order Diptera is a consequence of the fact that: (1) under specific temperature and weather conditions, they exhibit predictable growth rates that can be correlated to time of death or postmortem interval (PMI), and (2) in general, the predictable order in which insect species arrive (termed succession) is a function of the level of decomposition. ${ }^{1,3-6}$ The ability to infer this crucial information hinges on correct identification of the species associated with any insect-related evidence retrieved at or in the vicinity of the corpse. Members of the insect family Calliphoridae, which include most species of blow flies, are typically the earliest arrivers, often appearing within $\sim 5-15$ min after death. ${ }^{7}$ Flies at a distance of up to $1-2$ miles are attracted to the corpse via chemical and/or visual cues, and the corpse is used as a food resource for larvae (i.e., maggots) which emerge from hatched eggs. ${ }^{8,9}$ Because the adult flies disperse when the corpse is disturbed, the most valuable entomological evidence which can be retrieved for species identification is that left behind by female flies such as eggs, larvae, or the remnants of other life stages such as puparial casings. ${ }^{10}$ However, although each life stage is clearly distinguishable from the others, the same life stage of closely related species are often difficult to tell apart. For example, even to the trained eye, it is almost impossible to differentiate between the eggs of multiple fly species. ${ }^{11}$ Figure 1 illustrates the similarities between the eggs of four Calliphoridae species (Lucilia sericata, Phormia regina, Lucilia coeruleiviridis, and Calliphora vicina), and demonstrates that although differences between adult flies are visually more readily apparent, the eggs are not as easily distinguished. Thus, it remains common practice to rear the eggs to adulthood so that the macroscopic

Received: May 6, 2017

Accepted: June 6, 2017

Published: June 6, 2017 


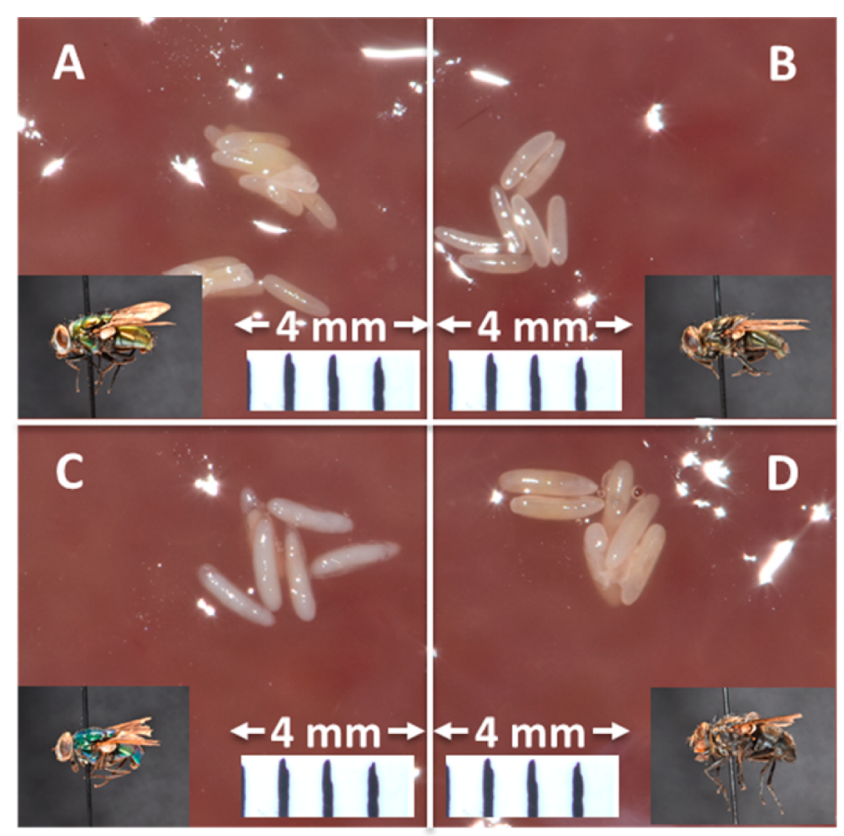

Figure 1. Images of eggs derived from four blow fly species from the Calliphoridae family, with the inset in each case showing a picture of the adult fly species corresponding to the eggs displayed in each panel. (A) Lucilia sericata eggs and adult fly; (B) Phormia regina eggs and adult fly; (C) L. coeruleiviridis eggs and adult fly; (D) Calliphora vicina eggs and adult fly. The scale provided refers to egg images only and does not reflect adult size.

features of the adult fly can be used for species identification. This is a time- and resource-intensive process which not only requires expert knowledge of rearing temperatures and optimal incubation periods, but also necessitates that the recovered eggs be viable. ${ }^{12}$ Importantly, eggs retrieved from corpses are often suspended in aqueous ethanol on-site, which precludes the possibility of rearing them to adulthood. ${ }^{13,14}$ Identification of larvae and pupae presents similar challenges. Their weights, dimensions, and morphological features can be compared with published standard charts to facilitate species identification and determination of possible age. ${ }^{15-17}$ However, this approach can yield inaccurate results not only due to a lack of reliable taxonomic keys, but also because of the common appearance of developmental variations that emerge as a consequence of differences in nutritional exposure or the presence of toxicological contaminants. ${ }^{18-20}$ Furthermore, the manner in which death occurred and the conditions of the corpse can greatly alter the size of the larvae, making accurate interpretation of their physical characteristics tenuous.

For these reasons, alternative species determination methods have been explored. Light and scanning electron microscopy with or without sample staining have been used to distinguish between eggs of different genera. ${ }^{21}$ However, it can be quite challenging to differentiate between the eggs of closely related species. ${ }^{22}$ Furthermore, identification by this approach relies on the existence of entomological keys to which the images can be compared, and these are often not available for a broad enough range of species. ${ }^{11}$ DNA barcoding using a $600 \mathrm{bp}$ fragment of the mitochondrial cytochrome $c$ oxidase gene to assign individuals to species is another method that is being developed. ${ }^{23}$ This approach is potentially very powerful because tissue from any available life stage can be used as source material. $^{24,25}$ In addition, insect life stage parts need not be intact, as is required for visual or microscopy-facilitated identification. ${ }^{24,26}$ However, patterns of mitochondrial variability can be confounded by the spread of maternally transmitted bacteria such as Wolbachia that cosegregate with mitochondria. ${ }^{23,27}$ This can result in low species identification success rates due to monophyly of many species at the mitochondrial level (i.e., different species will have identical barcodes). ${ }^{23,24}$ This approach also requires the development of comprehensive DNA barcodes for the hundreds of relevant species, which is time-consuming, labor-intensive, and has yet to be accomplished. ${ }^{26}$ An emerging technique for species identification is hydrocarbon profiling. Cuticular hydrocarbon composition varies as a function of age and species, and thus, chemical profiles can be used to discriminate between different species of insects. ${ }^{3,28}$ This approach has been applied successfully to distinguish between different species of African and European honeybees and pine engraver beetles. ${ }^{29-31}$ Hydrocarbon profiles of puparial casings have also been shown to correlate to species. ${ }^{32}$ In addition, it has been demonstrated that multivariate statistical analysis processing of these profiles can aid in species differentiation and confer an additional level of statistical certainty to the identification. ${ }^{32}$

It would be highly advantageous to be able to rapidly determine the species of entomological evidence representative of the earliest fly life stages, most notably the eggs, because this would circumvent the need for further time-consuming analyses associated with more commonly used conventional species identification methods. Desirable features of such a method would include avoiding the need for the eggs to be reared to adulthood, as well as the sample preparation steps required for microscopic analysis. The ability to directly analyze evidence in the form in which it is prepared at the crime scene would be an added benefit, as would the reporting of species identification confidence levels. Toward the goal of developing an approach that would fulfill the aforementioned requirements, we show here that the chemical profiles of ethanol solutions in which blow fly eggs are stored exhibit egg-derived chemical fingerprints that can be used to identify species. These signatures can be rapidly determined using direct analysis in real time-high resolution mass spectrometry (DART-HRMS), and chemometric processing of this data provides a means whereby species can be identified. We demonstrate proof-of-principle using eggs verified to be derived from four blow fly species (family Calliphoridae): Calliphora vicina (Robineau-Desvoidy), Lucilia sericata (Meigen), L. coeruleiviridis (Macquart), Phormia regina (Meigen), and members of the Phoridae and Sarcophagidae families.

\section{METHODS}

Chemical Standards. The following amino acid standards were purchased from Sigma-Aldrich (St. Louis, MO): arginine, glutamine, isoleucine, lysine, proline, and valine. Cysteine, glutamic acid, serine, and tyrosine were acquired from Oakwood Products (Estill, SC). The MALDI matrix 2,5dihydroxybenzoic acid (DHB) was purchased from Acros Organics (Morris Plains, NJ). The following solvents were purchased from the indicated vendors: acetonitrile, ethanol, and n-butanol (Fisher Scientific, Pittsburgh, PA); acetone (Pharmco-Aaper, Shelbyville, KY); trifluoroacetic acid (Acros Organics, Morris Plains, NJ); and acetic acid (Mallinckrodt, St. Louis, MO). Ninhydrin was purchased from Pierce Chemical (Rockford, IL) and silica gel IB-F thin layer chromatography 
plates $(2.75 \times 7.5 \mathrm{~cm})$ were purchased from J.T. Baker (Center Valley, PA).

Photographic Imaging. Adult specimens were obtained from the Forensic Entomology Insect Collection at John Jay College. Adult flies were pinned through the thorax using \#3 BioQuip insect pins (Rancho Dominguez, CA) and placed on a Styrofoam platform. Photographs were taken using a Nikon D5500 camera with a Nikon AF-S MicroNikkor $105 \mathrm{~mm}$ micro-lens. Egg specimens were obtained by placing $35 \mathrm{~g}$ of fresh pork liver into rearing colonies maintained by the Dr. Rosati Lab at John Jay College. Liver was placed into colony cages of L. sericata, P. regina and C. vicina where it remained for 16 h. Deposited eggs were collected. Eggs of L. coeruleiviridis were obtained from a preserved sample derived from a colony maintained during spring 2016. Fresh pork liver (35 g) was placed in the colony cage for approximately $12 \mathrm{~h}$, and eggs were collected and stored in $70 \%$ ethanol solution. Blow fly eggs (fresh and preserved) were placed on $35 \mathrm{~g}$ of fresh pork liver for imaging purposes. Images were taken using a Zeiss Discovery v8 Stereoscope with a 10x ocular lens, and a Plan S 1.0x FWD $81 \mathrm{~mm}$ objective lens set on a magnification of $1 \times$.

Collection and Preservation of Blow Fly Eggs. Stock blow fly colonies were maintained in the entomology lab of Dr. Jennifer Rosati (John Jay College of Criminal Justice, New York, NY). Adult flies were housed in $45 \times 45 \times 45 \mathrm{~cm}$ steel and mesh cages with a 12L:12D diel cycle, $50 \%$ humidity, and a temperature of $21{ }^{\circ} \mathrm{C} .{ }^{33}$ Adult flies were fed milk powder and sugar placed in a $100 \mathrm{~mm}$ plastic Petri dish (Fisher Scientific, Pittsburgh, PA) and water ad libitum in a $500 \mathrm{~mL}$ Erlenmeyer flask plugged with paper towels to prevent drowning. L. sericata and Sarcophagidae colonies were maintained since 2014; Phoridae colonies were started in 2015, and C. vicina and L. coeruleiviridis colonies began in 2016, all with flies collected from the Manhattan area. $P$. regina colonies were originally obtained from Dr. Christine Picard at Indiana UniversityPurdue University (Indianapolis) and subsequently established at John Jay College in 2015. All colonies are augmented annually with wild-type females collected from the Manhattan, NY area with traps baited with pork liver. For the collection of the fresh eggs used in these experiments, pork liver was used as an oviposition medium and was placed inside each colony cage until sufficient eggs (approximately 100) were collected. The eggs were then removed from the tissue and were suspended in $70 \%$ aqueous ethanol storage solutions. Approximately 10-50 eggs of a given species were placed into a vial containing $3 \mathrm{~mL}$ of solution. Five replicate vials were prepared for each of the four species of blow fly: C. vicina, L. sericata, L. coeruleiviridis, $P$. regina, and members of the Phoridae and Sarcophagidae families. The samples were stored at $4{ }^{\circ} \mathrm{C}$ until analysis.

DART-HRMS Experiments. Mass spectral data were acquired using a DART-SVP ion source (IonSense, Saugus, MA) attached to a JEOL AccuTOF mass spectrometer (JEOL USA, Peabody, MA) in positive-ion mode. The DART ion source grid voltage was $50 \mathrm{~V}$, and the gas heater temperature was set to $350{ }^{\circ} \mathrm{C}$. Mass spectrometer settings were: ring lens voltage, $5 \mathrm{~V}$; orifice 1 voltage, $20 \mathrm{~V}$; orifice 2 voltage, $5 \mathrm{~V}$; and peak voltage, $400 \mathrm{~V}$. The helium flow rate of the DART ion source was $2.0 \mathrm{~L} / \mathrm{min}$. Spectra were collected at 1 spectrum per $\mathrm{s}$ over the mass range $\mathrm{m} / z 40-800$. The resolving power of the mass spectrometer was 6000 FWHM. Mass calibration was performed using polyethylene glycol (PEG 600).

Ethanol suspensions of fly egg masses that were created by the addition of eggs to the aqueous ethanol storage solutions were analyzed by dipping the closed end of a melting point capillary tube into the solution and presenting the coated surface to the open-air space between the mass spectrometer inlet and ion source. A minimum of three replicates of each sample were analyzed and averaged together, and five samples of each species were tested to ensure a comprehensive data set. The chemical standards, amino acids, and calibrants, all of which were solids, were also analyzed by dipping the closed end of a melting point capillary tube into the powder and presenting it to the ion source.

In-source collision-induced dissociation (CID) by DARTHRMS was performed on ethanol egg suspension samples and standard solutions using function switching, where spectra are acquired with a predefined set of increasing orifice 1 voltages within a single MS experiment. In these analyses, the orifice 1 voltage was varied from $20,30,60$, and $90 \mathrm{~V}$, which caused increasing levels of fragmentation.

Data processing, including calibration, background subtraction, and peak centroiding, were performed using TSSPro3 software (Schrader Analytical Laboratories, Detroit, MI). Mass spectral analysis, elemental composition, and linear discriminant analysis (LDA) were conducted using Mass Mountaineer (Mass-spec-software.com, RBC Software, Portsmouth, NH).

Multivariate Statistical Analysis of DART-HRMSDerived Chemical Fingerprints. Multivariate statistical analysis processing was accomplished through linear discriminant analysis (LDA). The raw mass spectral data were used to generate principal components (PCs) based on peaks within a 7 millimass unit tolerance and above a threshold of $5 \%$ relative abundance. The LDA plot was created from the first three PCs, and evaluation of the classification system was performed by leave-one-out cross-validation (LOOCV). In this validation technique, each sample is excluded individually from the training set, which is then rebuilt, and then the excluded sample is tested as an unknown to determine the class to which it is assigned by the model. This process is repeated for each data point, and the strength of the model is assessed by the accuracy of the predicted classification assigned to each sample.

Amino Acid Identification by MALDI-MS/MS Analysis of Eggs. Matrix assisted laser desorption ionization-high resolution mass spectrometry (MALDI-HRMS) analyses were performed using a JMS-S3000 SpiralTOF MALDI TOF/TOF mass spectrometer (JEOL USA, Peabody, MA). Mass spectral data were recorded in positive spiral mode, and spectra were obtained in the range of $\mathrm{m} / z 50-1000$ at a sampling interval of 0.5 ns. The laser frequency was set to $250 \mathrm{~Hz}$. Several instrument parameters such as detector power, delay time, and laser intensity were optimized for each sample analysis. The delay time was set between 10 and $20 \mathrm{~ns}$; the detector power was generally between 60 and $70 \%$, and the laser intensity was $\sim 85 \%$. Data processing was performed using msTornado and msMicroImager (JEOL Ltd., Akishima, Tokyo, Japan) and included centroiding and peak selection as well as the compilation of ion image renders. Initial analyses of the amino acid standards and egg extract solutions were performed in spiral mode to determine the elemental composition of the amino acids present. Next, individual ions were selected for further MS/MS analysis to confirm the presence of individual amino acids.

The matrix solution used for analyses was comprised of 10 $\mathrm{mg} / \mathrm{mL} \mathrm{DHB}$ in $1: 1$ water:acetonitrile with $1 \%$ trifluoroacetic acid $(\mathrm{v} / \mathrm{v})$. Authentic amino acid standards were first analyzed for later comparison to egg extract analysis results. Standard 


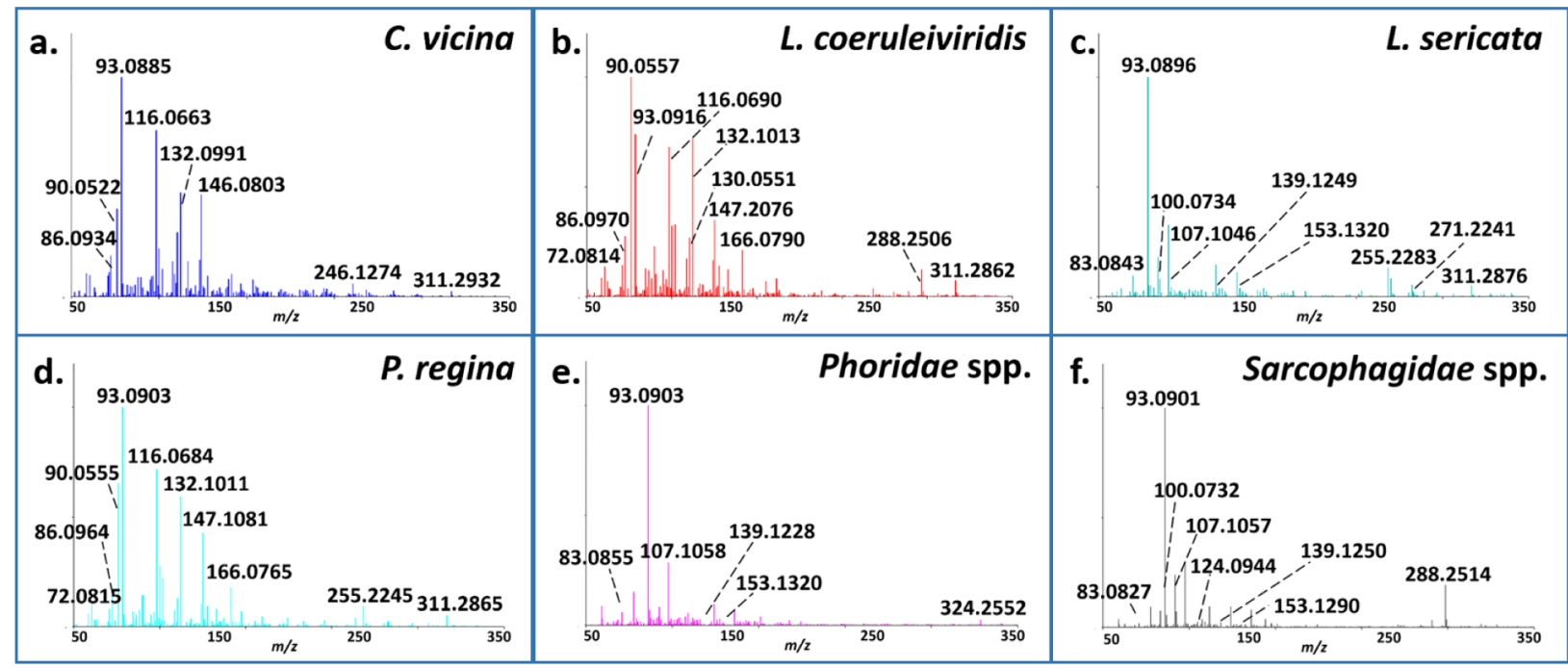

Figure 2. Results of DART-HRMS analyses of aqueous ethanol suspensions of six species of fly eggs: (a) C. vicina, (b) L. coeruleiviridis, (c) L. sericata, (d) P. regina, (e) Phoridae spp., and (f) Sarcophagidae spp. All analyses were performed in positive ion mode at $350{ }^{\circ} \mathrm{C}$. Each spectrum represents an average of three analyses. The spectra generated for each species are unique and can be used as fingerprints for the identification of individual species.

solutions comprised of $5 \mathrm{mg}$ of each amino acid in $0.5 \mathrm{~mL}$ of 1:1 water:acetonitrile with $1 \%$ trifluoroacetic acid (v/v) were prepared. For MALDI analysis, a mixture of $1 \mu \mathrm{L}$ of amino acid standard solution in $10 \mu \mathrm{L}$ of matrix solution was applied to a stainless steel target plate.

To detect and confirm the presence of amino acids in eggs, approximately $0.05 \mathrm{~g}$ of eggs was transferred to a $2.0 \mathrm{~mL}$ Eppendorf tube and ground into a paste using a metal spatula. Acetonitrile $(1 \mathrm{~mL})$ was added. The mixture was sonicated using a Bransonic B-3 Ultrasonic Cleaner (Branson Ultrasonics, Danbury, CT) for $30 \mathrm{~min}$ and then centrifuged. The supernatant was removed with a pipet and transferred to an Eppendorf tube, which was left uncapped for approximately 24 $\mathrm{h}$ to allow the solvent to evaporate completely. The resulting residue was then resuspended in $100 \mu \mathrm{L}$ of acetonitrile. This solution $(5 \mu \mathrm{L})$ was then analyzed by MALDI-SpiralTOF HRMS by first adding $6 \mu \mathrm{L}$ of the matrix solution and then transferring $0.5 \mu \mathrm{L}$ of the resulting mixture onto a stainless steel target plate. The external calibrant used for these experiments was prepared by adding $1 \mu \mathrm{L}$ of PEG 200 to $10 \mu \mathrm{L}$ of matrix solution. The amino acid standards, egg samples, and calibrant were applied to stainless steel target plates $(0.5 \mu \mathrm{L})$ and allowed to air-dry before MALDI-HRMS analysis.

Amino Acid Identification by Thin Layer Chromatography (TLC). Because alanine and glycine have masses below $100 \mathrm{Da}$, it was not possible to confirm their presence by MS/ MS using our MALDI-HRMS instrument. Therefore, to confirm the presence of these amino acids in the fly eggs, analysis by TLC was performed. Eggs $(\sim 0.1 \mathrm{~g})$ were placed into an Eppendorf tube and ground into a paste using a metal spatula. To this was added $500 \mu \mathrm{L}$ of the TLC elution solvent which was comprised of $n$-butanol, acetic acid, and water (3:1:1 $\mathrm{v} / \mathrm{v})$. The egg sample was then sonicated for $20 \mathrm{~min}$. Glycine and alanine standard solutions were prepared by adding $5 \mathrm{mg}$ of the amino acid to $1 \mathrm{~mL}$ of the TLC elution solvent. The standards and egg solutions were then spotted onto a TLC plate, which was developed in the TLC elution solvent for approximately $1.5 \mathrm{~h}$. After development was complete, the plate was removed from the TLC chamber and allowed to air-dry. A ninhydrin stain comprised of $0.1 \mathrm{~g}$ of ninhydrin dissolved in a solution of $0.5 \mathrm{~mL}$ acetic acid and $100 \mathrm{~mL}$ acetone was applied to the entire plate using a plastic spray bottle (CVS, Woonsocket, RI). The plate was again air-dried before it was placed in a $100{ }^{\circ} \mathrm{C}$ oven for $3 \mathrm{~min}$. During this time, colors indicative of the presence of each individual amino acid appeared. The $R_{\mathrm{f}}$ values of the spots representative of the amino acid standards and the amino acids in the eggs were calculated.

\section{RESULTS}

Necrophagous Fly Egg Species Identification and Differentiation. DART-HRMS analyses of ethanol samples in which blow fly eggs were suspended were performed in positive ion mode at $350{ }^{\circ} \mathrm{C}$. The results for the six fly species C. vicina, L. coeruleiviridis, L. sericata, P. regina, Phoridae, and Sarcophagidae are shown in Figure 2, and the related mass measurement data, including measured masses and relative peak abundances, are listed in Supporting Information Table S1. Visual inspection of the spectra showed that each species exhibited a unique chemical fingerprint. The spectra displayed between 21 and 90 peaks above a threshold of $2 \%$ relative abundance depending on the species. L. coeruleiviridis had the greatest number of peaks, and Sarcophagidae had the least.

Although the chemical profile of each species was unique, some peaks were common to all species, including nominal $\mathrm{m} / \mathrm{z}$ 93 and 100. Others were observed in most of the spectra such as $m / z 90$, which appeared in the spectra of every species except for Phoridae, and nominal $\mathrm{m} / z 72$ and 115 , which were observed in the spectra of all species except Sarcophagidae. A number of peaks were only seen in a small subset of spectra. For example, $m / z 76$ was observed only in $L$. coeruleiviridis, $C$. vicina, and P. regina; $m / z 288$ and 289 were detected only in $L$. coeruleiviridis and Sarcophagidae; and $m / z 246$ was seen only in C. vicina. Nominal $m / z 271$ was detected only in L. sericata and $P$. regina. There were a handful of peaks that were observed only in the four species C. vicina, L. coeruleiviridis, L. sericata, and $P$. regina. These included nominal $\mathrm{m} / z 311$. Additionally, peaks such as $m / \mathrm{z} 120,130$, and 146 appeared only in C. vicina, L. coeruleiviridis, and $P$. regina. 
From the high-resolution data, it was possible to make tentative structural assignments for several of the detected masses. A peak at nominal $\mathrm{m} / \mathrm{z} 93$ was observed in the spectra of all 6 ethanol egg suspensions and corresponded to the formula $\mathrm{C}_{4} \mathrm{H}_{12} \mathrm{O}_{2}+\mathrm{H}^{+}$or the protonated dimer of ethanol. Many of the observed peaks had formulas consistent with those of amino acids. The peak at nominal $\mathrm{m} / \mathrm{z} 116$, which was detected in the mass spectral profiles of $C$. vicina, $L$. coeruleiviridis, $P$. regina, L. sericata, and Sarcophagidae, corresponded to the formula $\mathrm{C}_{5} \mathrm{H}_{9} \mathrm{NO}_{2}+\mathrm{H}^{+}$and was tentatively identified as proline. A peak at $\mathrm{m} / z 90$ corresponding to $\mathrm{C}_{3} \mathrm{H}_{7} \mathrm{NO}_{2}+\mathrm{H}^{+}$was detected in the spectra of all of the species except Phoridae, and was assigned to alanine. Protonated forms of the isomeric amino acids isoleucine/leucine were also believed to be present in all of the ethanol egg suspensions except Phoridae, as a peak at nominal $\mathrm{m} / z 132$ was observed. The peak at $\mathrm{m} / z 118$ that was detected in the spectra of $C$. vicina, $L$. coeruleiviridis, $P$. regina, $L$. sericata, and Phoridae was assigned to protonated valine. Glycine $(m / z 76)$, glutamic acid $(m / z 148.08)$, phenylalanine $(\mathrm{m} / z 166)$, histidine $(\mathrm{m} / z 156)$, methionine $(\mathrm{m} / z 150)$, serine $(m / z 106)$, threonine $(m / z 120)$, and tyrosine $(m / z 182)$ were all tentatively identified in the mass spectra of $C$. vicina, $L$. coeruleiviridis, and P. regina. Glutamic acid was observed at $\mathrm{m} / \mathrm{z}$ 148 in C. vicina, L. coeruleiviridis, and P. regina. Glutamine and tryptophan were detected only in the ethanol suspensions of $P$. regina at masses $m / z 147.08$ and 205 , respectively. The amino acid lysine was tentatively identified in the spectra representing L. coeruleiviridis and $P$. regina as indicated by the presence of a peak at $m / z 147.11$. Masses consistent with the presence of the protonated forms of the amino acids arginine, asparagine, aspartic acid, and cysteine at nominal $\mathrm{m} / z$ 175, 133, 134, and 122 respectively, were not detected in the spectra of any of the ethanol suspensions.

Confirmation of the Presence of Tentatively Assigned Biomarkers and Amino Acids by DART-HRMS In-Source Collision-Induced Dissociation. The presence of specific compounds can be confirmed by DART-HRMS through comparison of the mass spectral fragmentation pattern observed under in-source CID conditions of an analyzed sample to that of an authentic chemical standard that has been analyzed in a similar manner. ${ }^{34}$ A low orifice 1 voltage (e.g., 20 $\mathrm{V})$ produces a spectrum containing unfragmented protonated precursor molecules. By increasing this voltage, fragmentation occurs, and the pattern observed can then serve as a unique fingerprint for a given compound. We used this technique to confirm our tentative structural assignments. To begin these analyses, the optimal orifice 1 voltage (i.e., one which resulted in fragmentation while retaining the protonated precursor) was determined. It was found to be $30 \mathrm{~V}$ for alanine, glutamine, tryptophan, tyrosine, and valine, and $60 \mathrm{~V}$ for all of the other amino acids. Next, each egg suspension and standard was analyzed by DART-HRMS at this new orifice 1 voltage, and the spectra of the samples were compared to those of the authentic chemical standards analyzed under similar conditions.

The presence of all 15 of the amino acids detected in $P$. regina under soft ionization DART-HRMS conditions was confirmed by in-source CID. A representative example of the type of data obtained from these experiments is presented in Figure 3 and Supporting Information Figures S1-S14 for P. regina. The head-to-tail plot shown for the amino acid proline in Figure 3 is representative, while similar plots corresponding to the other amino acids are presented in Supporting

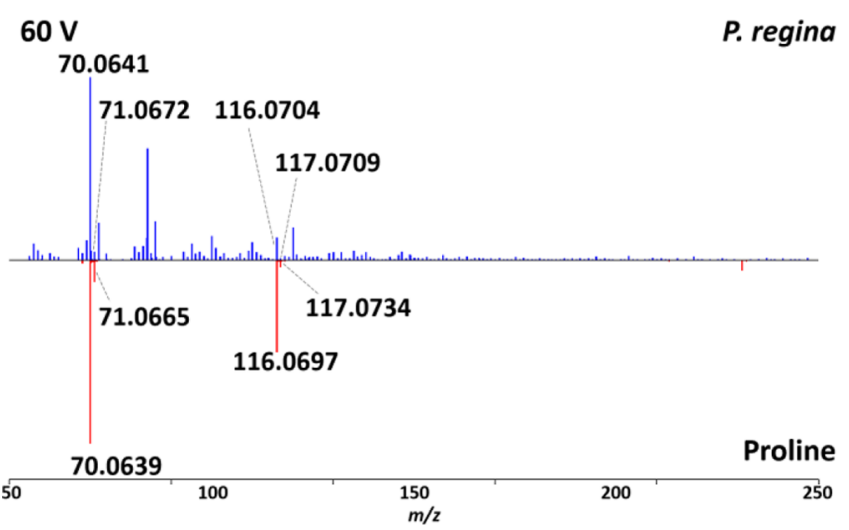

Figure 3. Head-to-tail plot showing the results of DART-HRMS insource collision-induced dissociation experiments performed to confirm the presence of proline in $P$. regina eggs. The top spectrum is of the $60 \mathrm{~V}$ experiment of the egg sample, and the bottom spectrum is that of the amino acid analyzed at the same voltage. The presence in the egg spectrum of the peaks that appear in the spectrum of the standard indicate that the proline is present in the blow fly eggs. Both the top and bottom spectra represent an average of three analyses.

Information Figures S1-S14. In Figure 3, the top spectrum shows the ethanol suspension of $P$. regina eggs analyzed at $60 \mathrm{~V}$, and the bottom spectrum is that of the proline standard analyzed under the same conditions. By this approach, the presence of proline in the eggs was confirmed through detection of not only the protonated precursor but also by detection of all of the fragment peaks that were seen for the proline standard. Thus, the fragment peaks observed at nominal $m / z 70,71,116$, and 117 from the proline standard were also observed in the spectrum for $P$. regina. By performing similar experiments with the other five species of fly eggs, the presence of various amino acids was confirmed (Supporting Information S1-S14). The profile of amino acids identified in each of the analyzed egg species is presented in Table 1 .

Independent Confirmation of the Presence of Amino Acids in Necrophagous Fly Eggs by MALDI-SpiralTOF HRMS. To confirm the DART-HRMS-facilitated amino acid assignments using an alternative method, samples were also analyzed by MALDI-MS/MS. As an example, the spectra obtained for $P$. regina eggs are presented in Supporting Information Figures S15-S29. Panel a of each image represents the results of the analysis of the egg sample, and panel b shows that of the authentic standard. By MALDI-SpiralTOF HRMS, high-resolution masses consistent with the amino acids arginine, asparagine, aspartic acid, glutamic acid, glutamine, histidine, isoleucine/leucine, lysine, methionine, phenylalanine, proline, serine, threonine, tryptophan, tyrosine, and valine were observed. The presence of alanine and glycine could not be confirmed by MALDI-MS/MS because their molecular weights are below $100 \mathrm{Da}$, the threshold for detection by the MALDISpiralTOF method. Of the subset of amino acids whose presence was confirmed by MALDI-SpiralTOF HRMS, arginine, asparagine, and aspartic acid were not detected in our DART-HRMS analysis of the blow fly egg ethanol suspensions. However, authentic standards of these amino acids were readily detected by DART-HRMS when analyzed individually.

Confirmation of the Presence of Amino Acids in Necrophagous Fly Eggs by TLC. While the presence of most of the amino acids in the fly eggs could be confirmed by DART- 
Table 1. Amino Acids Detected in Ethanol Suspensions of Six Species of Fly Egg Samples. Their presence was confirmed by comparing the fragmentation patterns of the in-source CID spectra of the egg suspensions to those of the authentic standards acquired under identical conditions.

\begin{tabular}{|c|c|c|c|c|c|c|}
\hline amino acid & C. vicina & L. coeruleiviridis & L. sericata & P. regina & Phoridae spp. & Sarcophagidae spp. \\
\hline alanine & $\mathrm{x}$ & $\mathrm{x}$ & $\mathrm{x}$ & $\mathrm{x}$ & & $\mathrm{x}$ \\
\hline \multicolumn{7}{|l|}{ arginine } \\
\hline \multicolumn{7}{|l|}{ asparagine } \\
\hline \multicolumn{7}{|l|}{ aspartic acid } \\
\hline \multicolumn{7}{|l|}{ cysteine } \\
\hline glutamine & & & & $\mathrm{x}$ & & \\
\hline glutamic acid & $\mathrm{x}$ & $\mathrm{x}$ & & $\mathrm{x}$ & & \\
\hline glycine & $\mathrm{x}$ & $\mathrm{x}$ & & $\mathrm{x}$ & & \\
\hline histidine & $\mathrm{x}$ & $\mathrm{x}$ & & $\mathrm{x}$ & & \\
\hline isoleucine/leucine & $\mathrm{x}$ & $\mathrm{x}$ & $\mathrm{x}$ & $\mathrm{x}$ & & $\mathrm{x}$ \\
\hline lysine & & $\mathrm{x}$ & & $\mathrm{x}$ & & \\
\hline methionine & $\mathrm{x}$ & $\mathrm{x}$ & & $\mathrm{x}$ & & \\
\hline phenylalanine & $\mathrm{x}$ & $\mathrm{x}$ & & $\mathrm{x}$ & & \\
\hline proline & $\mathrm{x}$ & $\mathrm{x}$ & $\mathrm{x}$ & $\mathrm{x}$ & & $\mathrm{x}$ \\
\hline serine & $\mathrm{x}$ & $\mathrm{x}$ & & $\mathrm{x}$ & & \\
\hline threonine & $\mathrm{x}$ & $\mathrm{x}$ & & $\mathrm{x}$ & & \\
\hline tryptophan & & & & $\mathrm{x}$ & & \\
\hline tyrosine & $\mathrm{x}$ & $\mathrm{x}$ & & $\mathrm{x}$ & & \\
\hline valine & $\mathrm{x}$ & $\mathrm{x}$ & $\mathrm{x}$ & $\mathrm{x}$ & $\mathrm{x}$ & \\
\hline
\end{tabular}

or MALDI-SpiralTOF HRMS, the presence of alanine and glycine was confirmed by TLC analysis of an egg paste extract (Supporting Information Figure S30). The $R_{\mathrm{f}}$ values of the spots observed for the alanine and glycine standards were 0.430 and 0.342 respectively. Six spots were observed in the TLC analysis of the egg sample with $R_{\mathrm{f}}$ values of $0.193,0.325,0.430$, $0.553,0.596$, and 0.640 . Spots with similar $R_{\mathrm{f}}$ values were observed in the analysis of the egg samples. The bands corresponding to alanine and glycine produce characteristic deep red and pale yellow colors, respectively, when stained with ninhydrin, and these same colors were observed for the TLC spots with the $R_{\mathrm{f}}$ values corresponding to glycine and alanine.

Multivariate Statistical Analysis of DART-HRMS Mass Spectral Fingerprints Enabled Species-Level Differentiation. The results of the DART-HRMS analyses of fly eggs revealed intraspecies similarities and interspecies differences, which could in principle be exploited for species identification purposes. To test this hypothesis, the DARTHRMS data were subjected to multivariate statistical analysis. The training set was comprised of 90 spectra in total. Linear discriminant analysis (LDA) was performed using the 15 masses shown in Table 2 . These $\mathrm{m} / z$ values represented peaks

Table 2. Feature Masses Used for the LDA Plot Presented in Figure 4

$\begin{array}{lll}90.0557 & 127.1124 & 171.1347 \\ 94.0953 & 132.0983 & 191.1561 \\ 100.0734 & 146.0794 & 255.2207 \\ 116.0669 & 147.1076 & 311.2935 \\ 118.0840 & 166.0790 & 324.2552\end{array}$

that most species had in common (e.g. nominal $\mathrm{m} / z$ 90, 116, and 132 that were observed in all species except Phoridae, $\mathrm{m} / z$ 118 and 171 that were detected in all species except Sarcophagidae, and $m / z 94$ and 100 which were detected in all species); $m / z \quad 146$ and 166 observed in C. vicina, $L$. coeruleiviridis and $P$. regina; $m / z 127$ detected in $L$. coeruleiviridis, L. sericata and Phoridae; $\mathrm{m} / z 147$ detected in $L$. coeruleiviridis and $P$. regina; $m / z 191$ only detected in $L$. coeruleiviridis and L. sericata; $m / z 255$ and 311 seen in C. vicina, L. coeruleiviridis, L. sericata, and P. regina; and $m / z 324$ only observed in Phoridae). The leave-one-out cross-validation (LOOCV) was $84 \%$, and $93 \%$ of the variance was accounted for by three principal components. Figure 4 shows two orientations of the LDA plot. Clustering of the data points representing the same species was observed.

\section{DISCUSSION}

The forensic value of juvenile life stages of forensically important fly families such as the eggs would be dramatically enhanced if their species identity could be more readily determined. We demonstrate here that the identification of species belonging to the Calliphoridae, Sarcophagidae, and Phoridae families can be rapidly accomplished through chemometric processing of DART-HRMS-derived chemical fingerprints of ethanol suspensions of eggs. Importantly, the DART-HRMS data is generated from samples of the type typically generated in the field (i.e., suspensions of eggs in aqueous ethanol). Because the ethanol samples are analyzed directly, none of the sample pretreatment steps that are essential for species identification by conventional methods are required. Our mass spectral analyses revealed a number of interesting trends, including: (1) The ethanol suspension of each species exhibited a chemical fingerprint that was remarkably consistent from sample to sample. (2) Chemical fingerprints differed between species, as illustrated by visual inspection of their mass spectra. (3) A subset of peaks was observed only in the mass spectra of C. vicina, L. coeruleiviridis, $L$. sericata, and $P$. regina, indicating the ability to distinguish between families as well as species. (4) The intraspecies similarities and interspecies differences of the chemical fingerprints were primarily a consequence of the variations in amino acid profiles of the ethanol suspensions. (5) Cysteine was not detected in any of the ethanol suspensions or the fly paste extracts. (6) DART-HRMS analysis of the ethanol suspension was superior to MALDI-HRMS analysis in that the 

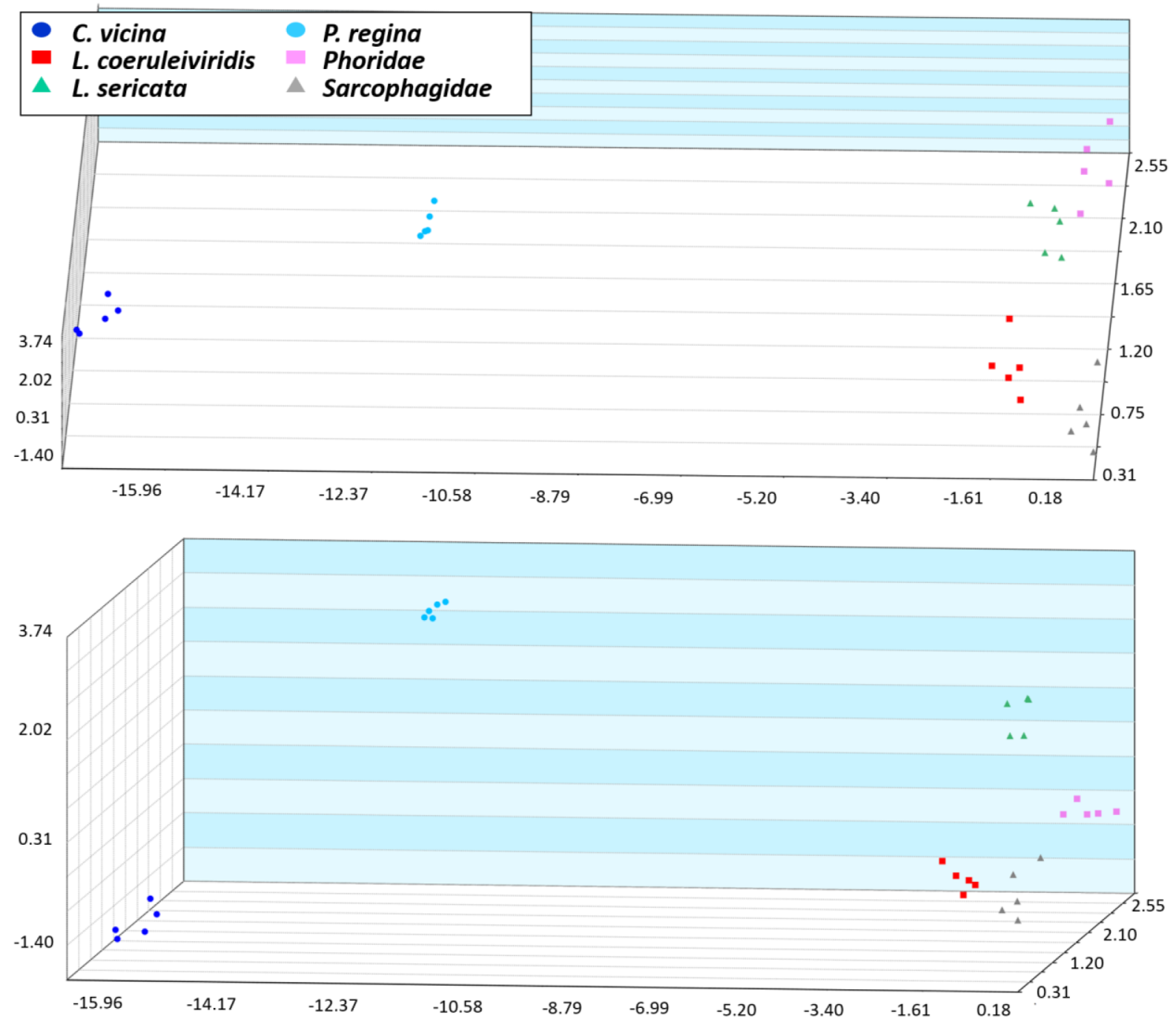

Figure 4. Two orientations of the results of the linear discriminant analysis (LDA) plot derived from a subset of the $m / z$ values observed in the mass spectra of ethanol suspensions of fly eggs representing the six species indicated. The data set included 90 mass spectral replicates, and 15 feature masses were used (listed in Table 2). These included some that were common to all species and others that were unique to individual species. Three principal components accounted for $93 \%$ of the variance, and the leave-one-out cross-validation (LOOCV) was $84 \%$. The plot shows tight clustering of the data points representing samples from the same species, and clear separation between those of different species.

former revealed a broader range of molecules, including amino acids. Detection of amino acids by MALDI-HRMS was only possible using concentrated extracts made from egg paste. The amino acids arginine, asparagine, and aspartic acid were absent in the DART-HRMS spectra of all six species of fly eggs despite the fact that the chemical standards were easily detected when analyzed by this method. This implies that our inability to observe these compounds in the ethanol whole egg suspensions may be that their levels were too low or because they did not leach into the ethanol/water solution from the eggs. This conclusion is supported by the fact that these amino acids were also not detected in MALDI-SpiralTOF HRMS analyses of the ethanol egg suspensions, but were observed only in samples prepared from macerated eggs (Supporting Information Figures S14-S16). The significance of the absence of cysteine in any of the egg samples is unknown.

It was the consistency of the intraspecies similarities and interspecies differences of the DART-HRMS fingerprints that enabled differentiation and species identification by chemometric processing of the DART-HRMS data. This is illustrated in the LDA plot. An implication of this result is that a DARTHRMS database of fly egg chemical signatures can be used to screen DART-HRMS chemical profiles of fly egg ethanol suspensions collected in the field, and that the results can be used for rapid species identification. To accomplish this, it will be necessary to acquire a comprehensive data set of DARTHRMS spectra representing multiple blow fly and other forensically relevant fly species. However, the speed with which the analyses can be performed (i.e., $\sim 3$ s per analysis) makes the generation of the multiple replicates required quite feasible. Furthermore, the data can be readily expanded to include an even larger number of species. The approach to species determination that is described here provides solutions to some of the challenges of more conventional methods. No sample preparation steps or specialized expertise is required. Furthermore, samples in the form in which they are prepared in the field can be analyzed. The extent to which intraspecies similarities and interspecies differences in chemical fingerprints occurs across the Diptera order is the subject of continuing investigations in our laboratories. 


\section{CONCLUSIONS}

The technique outlined here is the first demonstration of a rapid chemical fingerprint-based method for species identification of necrophagous fly eggs. The samples were analyzed by DART-HRMS in the form that they are typically collected at a crime scene without any further sample preparation. The analyses revealed that blow fly eggs exhibit species-specific differences in amino acid profiles. The DART-HRMS-chemometric data processing species identification approach circumvents many of the shortcomings of traditional analysis methods, including the time-intensive process of rearing eggs to adulthood to confirm species identity. The chemical profiles revealed intraspecies similarities and interspecies differences that served as the basis for chemometric analysis. The results show the application of DART-HRMS for the generation of unique species-specific chemical fingerprints that can be used to rapidly identify early life stage entomological evidence.

\section{ASSOCIATED CONTENT}

\section{S Supporting Information}

The Supporting Information is available free of charge on the ACS Publications website at DOI: 10.1021/acs.analchem.7b01708.

DART-HRMS in-source CID spectral data of aqueous ethanol egg extracts and amino acid standards, MALDIMS/MS spectral data of fly eggs, TLC analysis results, and a table of mass measurement data of fly egg spectra (PDF)

\section{AUTHOR INFORMATION}

\section{Corresponding Author}

*E-mail: rmusah@albany.edu.

\section{ORCID}

Rabi A. Musah: 0000-0002-3135-4130

\section{Author Contributions}

R.A.M. and J.Y.R. conceived the project. J.E.G. and J.Y.R. performed experiments. R.A.M. interpreted the results and wrote the manuscript. J.E.G. contributed to writing the manuscript. J.Y.R. reared and maintained fly colonies, collected eggs in the field, and conducted fly species identification. C.M.L. performed MALDI-SpiralTOF experiments.

\section{Notes}

The authors declare no competing financial interest.

\section{ACKNOWLEDGMENTS}

The authors gratefully acknowledge Kristen Fowble for assistance with MALDI-SpiralTOF HRMS experiments, Joey Fragale and Veena Mehta for insect care, Dr. Robert Cody for helpful discussions, and JEOL USA Inc. for the use of the JMSS3000 SpiralTOF MALDI TOF/TOF mass spectrometer. The financial support of the Research Foundation of SUNY is gratefully acknowledged.

\section{REFERENCES}

(1) Tabor, K. L.; Brewster, C. C.; Fell, R. D. J. Med. Entomol. 2004, 41, 785-795.

(2) Tabor, K. L.; Fell, R. D.; Brewster, C. C. Forensic Sci. Int. 2005, 150, 73-80.

(3) Pechal, J. L.; Moore, H.; Drijfhout, F.; Benbow, M. E. Forensic Sci. Int. 2014, 245, 65-71.

(4) Matuszewski, S.; Bajerlein, D.; Konwerski, S.; Szpila, K. Forensic Sci. Int. 2011, 207, 150-163.
(5) Matuszewski, S.; Bajerlein, D.; Konwerski, S.; Szpila, K. Forensic Sci. Int. 2010, 194, 85-93.

(6) Matuszewski, S.; Bajerlein, D.; Konwerski, S.; Szpila, K. Forensic Sci. Int. 2010, 195, 42-51.

(7) Vasconcelos, S. D.; Soares, T. F.; Costa, D. L. Int. J. Legal Med. 2014, 128, 229-233.

(8) Byrd, J. H.; Castner, J. L. Forensic Entomology: The Utility of Arthropods in Legal Investigations; CRC Press: Boca Raton, FL, 2002; p 681.

(9) Greenberg, J. A Natural History of the Chicago Region, 2nd ed.; University of Chicago Press: Chicago, IL, 2002.

(10) Sukontason, K.; Sribanditmongkol, P.; Ngoen-klan, R.; Klongklaew, T.; Moophayak, K.; Sukontason, K. L. Parasitol. Res. 2010, 106, 641-646.

(11) Greenberg, B.; Szyska, M. L. Ann. Entomol. Soc. Am. 1984, 77, $488-517$.

(12) Bourel, B.; Callet, B.; Hedouin, V.; Gosset, D. Forensic Sci. Int. 2003, 135, 27-34.

(13) Lopez-Esclapez, R.; Garcia, M. D.; Arnaldos, M. I.; Presa, J. J.; Ubero-Pascal, N. Micron 2014, 62, 43-51.

(14) Adams, Z. J.; Hall, M. J. Forensic Sci. Int. 2003, 138, 50-61.

(15) Day, D. M.; Wallman, J. F. Forensic Sci. Int. 2006, 159, 158-167.

(16) Sharma, R.; Kumar Garg, R.; Gaur, J. R. Egyptian J. of Forensic Sci. 2015, 5, 1-12.

(17) Wells, J.; LaMotte, L. J. Forensic Sci. 1995, 40, 585-590.

(18) Carvalho, L. M. L.; Linhares, A. X.; Trigo, J. R. Forensic Sci. Int. 2001, 120, 140-144.

(19) de Carvalho, L. M. L.; Linhares, A. X.; Badan Palhares, F. A. Forensic Sci. Int. 2012, 220, 27-32.

(20) Mai, M.; Amendt, J. Forensic Sci. Int. 2012, 221, 65-69.

(21) Sukontason, K.; Sukontason, K. L.; Piangjai, S.; Boonchu, N.; Kurahashi, H.; Hope, M.; Olson, J. K. Micron 2004, 35, 391-395.

(22) Sukontason, K.; Sukontason, K. L.; Piangjai, S. Rev. Inst. Med. Trop. Sao Paulo 2003, 45, 95-98.

(23) Whitworth, T. L.; Dawson, R. D.; Magalon, H.; Baudry, E. Proc. R. Soc. London, Ser. B 2007, 274, 1731-1739.

(24) Chen, W. Y.; Hung, T. H.; Shiao, S. F. J. Med. Entomol. 2004, 41, 47-57.

(25) Chen, M. S.; Wheeler, S.; Davis, H.; Whitworth, R. J.; Knutson, A.; Giles, K. L.; Royer, T. A.; Skinner, M. J. Econ. Entomol. 2014, 107, 1110-1117.

(26) Wells, J. D.; Sperling, F. A. Forensic Sci. Int. 2001, 120, 110-115.

(27) Avtzis, D. N.; Doudoumis, V.; Bourtzis, K. PLoS One 2014, 9, e112795.

(28) Zhu, G. H.; Xu, X. H.; Yu, X. J.; Zhang, Y.; Wang, J. F. Forensic Sci. Int. 2007, 169, 1-5.

(29) Lavine, B. K.; Vora, M. N. J. Chromatogr A 2005, 1096, 69-75.

(30) Ye, G.; Li, K.; Zhu, J.; Zhu, G.; Hu, C. J. Med. Entomol. 2007, 44, $450-456$.

(31) Page, M.; Nelson, L. J.; Blomquist, G. J.; Seybold, S. J. J. Chem. Ecol. 1997, 23, 1053-1099.

(32) Musah, R. A.; Espinoza, E. O.; Cody, R. B.; Lesiak, A. D.; Christensen, E. D.; Moore, H. E.; Maleknia, S.; Drijfhout, F. P. Sci. Rep. 2015, 5, 11520.

(33) Rosati, J. Y. Spatial and Temporal Variability in the Carrion Insect Community: Using Blow Flies (Family: Calliphoridae) as a Model System to Study Coexistence Mechanisms at Multiple Scales. University of Windsor, Electronic Theses and Dissertations, 2014.

(34) Lesiak, A. D.; Cody, R. B.; Dane, A. J.; Musah, R. A. Anal. Chem. 2015, 87, 8748-8757. 